\title{
Chaotic fluctuations in graphs with amplification
}

\author{
Stefano Lepria,b \\ ${ }^{a}$ Consiglio Nazionale delle Ricerche, Istituto dei Sistemi Complessi, Via Madonna del Piano 10 I-50019 Sesto Fiorentino, Italy \\ ${ }^{b}$ Istituto Nazionale di Fisica Nucleare, Sezione di Firenze, via G. Sansone 1 I-50019, Sesto Fiorentino, Italy
}

\begin{abstract}
We consider a model for chaotic diffusion with amplification on graphs associated with piecewise-linear maps of the interval. We investigate the possibility of having power-law tails in the invariant measure by approximate solution of the Perron-Frobenius equation and discuss the connection with the generalized Lyapunov exponents $L(q)$. We then consider the case of open maps where trajectories escape and demonstrate that stationary power-law distributions occur ,when $L(q)=r$, with $r$ being the escape rate. The proposed system is a toy model for coupled active chaotic cavities or lasing networks and allows to elucidate in a simple mathematical framework the conditions for observing Lévy statistical regimes and chaotic intermittency in such systems.
\end{abstract}

Keywords: Chaotic map, Power-law distributions, Diffusion and amplification on graphs, Generalized Lyapunov 'exponents

\section{Introduction}

Dynamical systems defined on graphs are subject of current research, due to the many applications to model complex interacting units with non uniform connectivity [1]. Also, one of the features of complex systems is the possibility of display non-Gaussian fluctuations that make large rare events very relevant. The distributions of the observables can have fat-tailed statistics leading to domination of a single event and lack of self-averaging of measurements. In active systems where fluctuating amplification can occur, even rare trajectories can generate large-sized fluctuations. This is well-known for multiplicative stochastic processes 2] and chaotic dynamical systems that display intermittency and multifractality [3].

Countless example are present in the physical, biological and even social sciences. A case of experimental relevance is provided by optical media with diffusion and amplification of light, as it occurs in random lasers where heavy-tailed distributions of emission intensities, characterized by Lévy -stable statistics [4] have been predicted [5] (see also [6, 7]) and confirmed in experiments [8, 9, 10].

An experimental system that encompasses properties of a dynamical systems on graphs and non trivial statistics is the lasing network, recently introduced in [11]. It consists of active and passive optical fibers, connected to form a graph structure. The connectivity induces a form of topological disorder and can be viewed as a discrete random laser, with a controllable complexity. The presence of the optical gain and disorder induce wild emission fluctuations whose origin is not fully understood [11]. So a related question is how such fluctuations relate to the network structure and connectivity.
The existence of fat tails is intimately related to the the possibility for a spontaneous fluctuation to grow well beyond the average. The indicators to quantify this are the finite-time and generalized Lyapunov exponents [12]. For multiplicative noise they been shown that they are useful tools to yield an intuitive derivation of the form of the probability distribution's tail in the presence of additive noise [13].

Another possibility to have stationary fat-tailed statistics for multiplicative growing processes is to consider resetting, namely a random process where the variable is set to a given value with some given protocol 14]. The cases of stochastic partial differential equations like the KardarParisi-Zhang equation of fluctuating interfaces has been als considered [15].

In the present paper we study a simple chaotic map that couples chaotic diffusion and random amplification. Nonlinear maps are thoroughly investigated as mathematically simple model to analyze the connection between macrolaws and microscopic chaos [16]. It can be regarded as a toy model for coupled active chaotic cavities or the lasing networks mentioned above [1]. The idea is that light rays can be treated as particles undergoing chaotic diffusion and amplification. Indeed, the classical dynamics of particles on graphs is a chaotic type of diffusive process [17]. Trajectories of a particle on a graph, undergoing scattering at its vertices, are in one-to-one correspondence with the ones of one-dimensional piecewise chaotic maps [17, 18].

The model is simple enough to allow for a very detailed analysis, demonstrating power-law distributions of the invariant measure. It allows to elucidate in a simple mathematical framework the conditions for observing 
Lévy statistical regimes and chaotic intermittency in such systems. It also serves as an example to demonstrate the usefulness of generalized Lyapunov exponents to assess the possibility of power-law fluctuations. Moreover we extend the concepts to the case of open systems (like chaotic repellors), a case that, to our knowledge, has not been studied in this terms.

In Section 2 the map model is presented and its relation with the physical systems is sketched. The stationary invariant measure is computed along with an effective master equation. The connection between power-law and generalized Lyapunov exponents is discussed in Section 3. This relation is extended to the case of open maps in Section 4. The connection between the model and the calculation of the spectrum of the lasing network is given in the Appendix.

\section{Map model}

We consider the following map

$$
\left\{\begin{array}{l}
x_{n+1}=f\left(x_{n}\right) \\
E_{n+1}=g\left(x_{n}\right) E_{n}+s
\end{array}\right.
$$

where $f$ is chaotic with a positive Lyapunov exponent $\lambda_{1}$. For definiteness, let us consider $x$ to belong to the unit interval and $E_{n}, g$ positive and $s \geq 0$ and small.

As suggested by the notation, one can imagine $x_{n}$ to describe couples the position of a "ray" undergoing chaotic motion during which it acquires an "energy" that increases or decreases according to whether $g$ il larger or smaller than one. Thus chaotic diffusion and amplification are coupled since the acquired energy depends on the trajectory. In Fig 1 we sketch a physical reference system inspired from the lasing network experimentally studied in [11, 19].

The term $s$ represent some form of energy injection and is needed to avoid that $E_{n}=0$ is not and "absorbing" point. The case $s=0$ leads to a non-stationary distribution that for large times is log-normal. This is readily understood as the variable $\log E_{n}=z_{n}$ performs a discrete-time biased random walk [20]. The average velocity $\left\langle\log g\left(x_{n}\right)\right\rangle \equiv \lambda_{2}$ is the (second) Lyapunov exponent of (11). The situation is drastically different in presence of a term $s>0$, that act as a source term. If $\lambda_{2}<0$ the variable $z_{n}$ is attracted towards the source and this yield a stationary measure. In the stochastic case this mechanism of repulsion has been shown to generically yield powerlaw decaying stationary distributions [21, 22, 23]. Similar considerations apply for extended stochastic systems like the non-linear diffusion equation with multiplicative noise [24]. On the other hand, for $\lambda_{2}>0$ the variable grows and some form of saturating mechanism is needed to ensure unbounded motion (more on this below).

To keep the analysis as simple as possible we consider the case of the piecewise-linear map

$$
f(x)= \begin{cases}\frac{1}{p} x & 0 \leq x \leq p / 2 \\ \frac{1}{1-p} x+\frac{1-2 p}{2(1-p)} & p / 2<x \leq 1 / 2 \\ \frac{1}{1-p} x-\frac{1}{2(1-p)} & 1 / 2<x \leq 1-p / 2 \\ \frac{1}{p} x+1-1 / p & 1-p / 2<x \leq 1\end{cases}
$$

see Fig,1. If we consider the motion of a particles on the graph there drawn, $f$ can be derived exactly as a suitable Poincaré section as described in [17]. 1 The map is everywhere expanding and is invariant for $x \rightarrow 1-x$, but it is straightforward to generalize to an asymmetric case and/or more complex graphs.

Since the invariant measure of the map is constant its the Lyapunov exponent $\lambda_{1}=-p \log p-(1-p) \log (1-p)$. So $\lambda_{1}>0$ but it is vanishingly small for $p$ approaching 0 and 1 where the map has weakly unstable orbits. Also, let us consider a piece-wise constant gain function $g$

$$
g\left(x_{n}\right)= \begin{cases}g & 0<x_{n} \leq \frac{1}{2} \\ l & \frac{1}{2}<x_{n}<1\end{cases}
$$

where $g \geq 1$ e $0<l \leq 1$. This is merely a choice of simplicity and it entails that the sequence of multipliers $g\left(x_{n}\right)$ is in one-to-one correspondence with symbolic dynamics of thomon $f$ Fron in thic cimnln nunmuln for $n+1 / 9$ thn
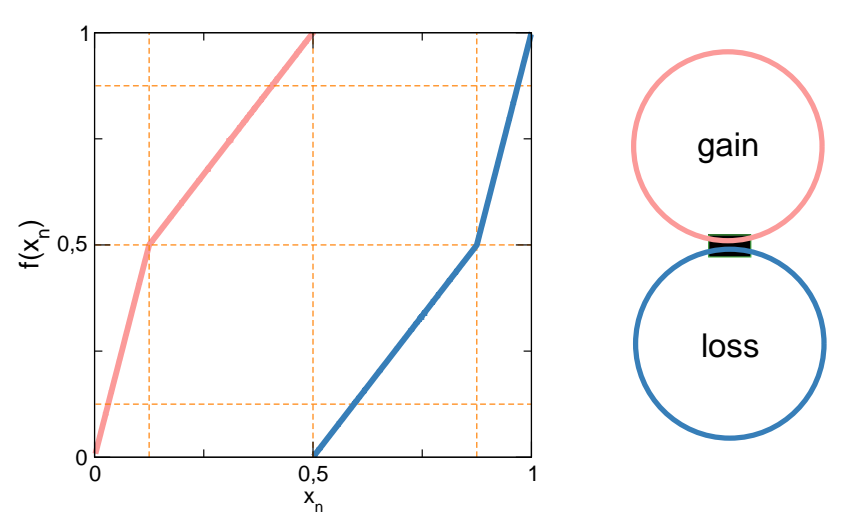

Figure 1: Left: the chaotic map (2) for $p=0.25$. Right: a sketch of two coupled chaotic cavities as in a double-ring lasing network connected by a coupler that transmits with a given probability (no reflections). One cavity contains an active amplifying medium, the other is dissipative. In the graph interpretation, the map $f$ can be derived exactly as a suitable Poincaré section as done in [17].

Let us focus on the case where the solution does not diverge namely $\langle\log g(x)\rangle<0$. Before entering the mathematical analysis, in Fig 2 we report some representative

\footnotetext{
${ }^{1}$ Thus the "physical" time $t_{n}$ corresponding to the $n$th iteration of the map and depends on the length on each bond. For instance, for the graph in Fig[1 with bond lengths $L_{1}$ and $L_{2}, t_{n+1}=t_{n}+$ $T\left(x_{n}\right) T\left(x_{n}\right)=T_{1}$ or $T_{2}$ for $x_{n}<1 / 2$ and $x_{n}>1 / 2$ respectively $(v$ being the particle velocity, $\left.T_{1,2}=L_{1,2} / v\right)$. This description can be generalized to arbitrary graphs associated with Markov dynamics, see [17].
} 

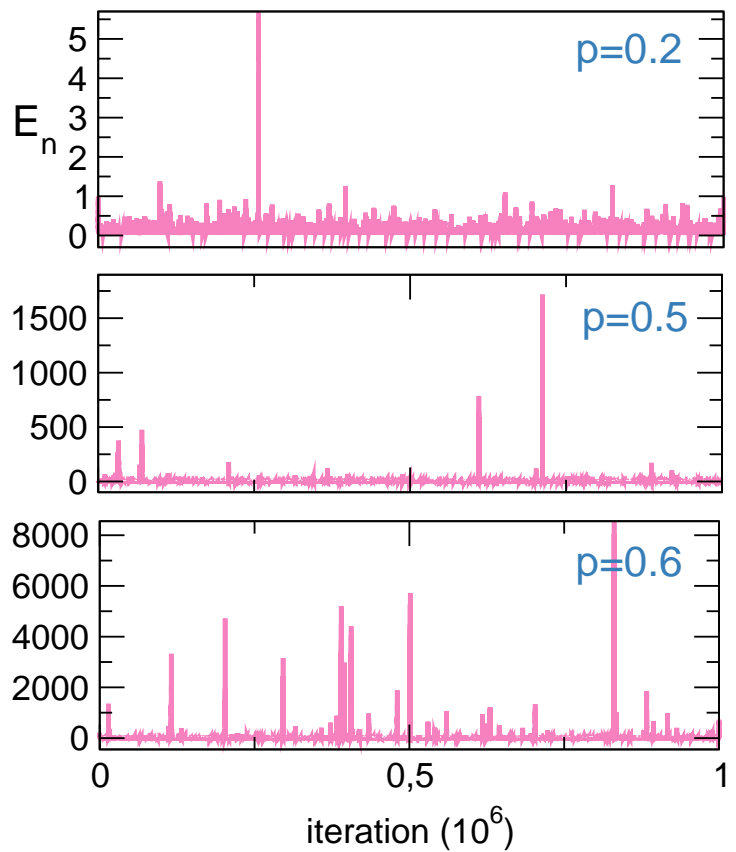

Figure 2: Time series of $E_{n}, g=1.2 l=0.8 s=10^{-3}$ for different values of $p$. The trajectory is highly intermittent with large excursion of short duration.

time-series of the the variable $E_{n}$. The dynamics is highly intermittent with large-amplitude spikes lasting tenths of iterates. A finite value of the source term insures that the variable does not vanish at long times, according to the mechanism mentioned above.

We are interested in the statistics of the variable $E_{n}$. The time evolution of the measure $P_{n}(x, E)$ can be computed as solution of Perron-Frobenius operator

$$
\begin{gathered}
P_{n+1}(x, E)= \\
= \begin{cases}\frac{p}{g} P_{n}\left(y_{1}, \frac{E-s}{g}\right)+\frac{(1-p)}{l} P_{n}\left(y_{2}, \frac{E-s}{l}\right) & 0 \leq x \leq \frac{1}{2} \\
\frac{(1-p)}{g} P_{n}\left(y_{3}, \frac{E-s}{g}\right)+\frac{p}{l} P_{n}\left(y_{4}, \frac{E-s}{l}\right) & \frac{1}{2}<x \leq 1\end{cases}
\end{gathered}
$$

where $y_{1}=p x, y_{2}=(1-p) x+\frac{1}{2}, y_{3}=(1-p) x+\frac{2 p-1}{2}$ and $y_{4}=p x+1-p$ are the preimages of $x$.

As a general approach, one may consider expanding $P_{n}$ on the basis of the eigen-functions of the Frobenius-Perron operator of the map $f$. This would allow to describe the full evolution of the measure in time, including transients associated with possibly slow chaotic diffusion. Since we are mostly interested in steady-state results, let us look for piecewise-constant in $x$ solutions of the form $P_{n}(x, E)=$ $P_{1, n}(E)$ for $0<x<1 / 2$ and $P_{n}(x, E)=P_{2, n}(E)$ for $1 / 2 \leq x<1$ respectively. We obtain

$$
\begin{aligned}
& P_{1, n+1}(E)=\frac{p}{g} P_{1, n}\left(\frac{E-s}{g}\right)+\frac{1-p}{l} P_{2, n}\left(\frac{E-s}{l}\right) \\
& P_{2, n+1}(E)=\frac{(1-p)}{g} P_{1, n}\left(\frac{E-s}{g}\right)+\frac{p}{l} P_{2, n}\left(\frac{E-s}{l}\right)
\end{aligned}
$$

We expect that this equation is valid when chaotic diffusion is sufficiently rapid to ensure homogeneization of the measure on the time scale faster than the typical growth. It has a form of a master equation for probabilities of the variable $E$ on each side of the interval. Also, a standard Kramers-Moyal expansion may be used to show that it corresponds to a set of coupled Langevin equations with multiplicative noise for the energies on the two sides of the interval. The connection between this equation and the one used in the calculation of the spectrum of the lasing network is given in the Appendix.

If we look for stationary solutions that decay as a power law for large $E \gg s$,

$$
P_{1,2}(E) \propto A_{1,2} E^{-(1+\alpha)}
$$

where $A_{1,2}$ are constants, the dependence on $s$ can be neglected and we get the self-consistency condition

$$
p\left(g^{\alpha}+l^{\alpha}\right)-(2 p-1) g^{\alpha} l^{\alpha}=1
$$

The latter, along with the the stability condition $\lambda_{2}<0$ i.e. $l g \leq 1$ determines the region of validity of the powerlaw solution (6). In particular, the more interesting case is when $\alpha<2$ yielding distributions with diverging variance like in the well-know case of Lévy stable distributions [4].

Although the source term $s$ is essential to yield a stationary distribution, its value does enter in the exponent of asymptotic decay (5). We also checked numerically that basically the same statistics if found if $s$ is replaced by a random positive number (additive noise).

To emphasize the importance of the separation of time scales leading to equations (4) and thus to power-laws let us compare with a situation when chaotic diffusion is relatively slow. For instance, in Fig 3 we consider the case $p$ is small. In this limit, the map has a weakly unstable period two orbit and $\lambda_{1} \approx p$. Not surprisingly, the invariant measure is non uniform and fractal in the direction of the variable $z$.

\section{Generalized Lyapunov exponents}

The generalized Lyapunov exponents $L(q)$ define the growth of the $q$ th moment of the perturbation [25, 3, 26, 27]. In general, for a perturbation $\delta u$ of a dynamical system, which evolves according to the linearized equation of motion, let $R(\tau)=\|\delta u(t+\tau)\| /\|\delta u(t)\|$ be the response function after a time $\tau$ to a disturbance at time $t$. Then, for large times $\overline{R^{q}(\tau)} \sim \exp (L(q) \tau)$ where the overline denote a time average. If $L(q)>0$ for large enough $q$ then there is a finite probability that a small perturbation grow very large. Moreover, the deviation of $L(q)$ from a linear behavior in $q$ signal an intermittent dynamics [12].

The condition for power-law stationary tails can be obtained from generalized Lyapunov exponents [13]. In the present case we are interested in the generalized exponents associated with the $E_{n}$ variable when no source term is 


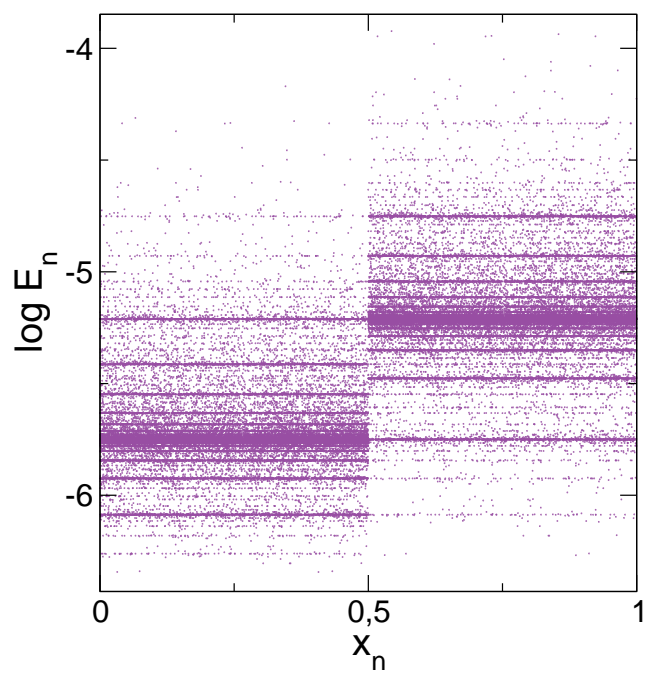

Figure 3: Iterates of the map $x_{n}, \log E_{n}, g=1.4 l=0.4$ s for $p=0.05$, yielding $\lambda_{1} \approx 0.05, \lambda_{2}=-0.290$ giving a $\mathrm{I}$ (Kaplan-Yorke) dimension 1.17.

present and unbounded growth or decay at large possible. Actually, using equations (41) with $s=0$ write the evolution map for the moments $\left\langle E_{1,2}^{q}\right\rangle$ anc $L$ as the logarithm of its largest eigenvalue,

$$
L(q)=\log \mid \frac{p\left(g^{q}+l^{q}\right)+\sqrt{p^{2}\left(g^{q}+l^{q}\right)^{2}-4(2 p-1}}{2}
$$

In the simplest case $p=1 / 2$ multipliers are unco] and one indeed gets the value $L(q)=\log \left(\frac{g^{q}+l^{q}}{2}\right)$ moment multiplier is just the arithmetic average $o$ $g^{q}$. Note that, by construction, the standard Ly exponent $\lambda_{2}=L^{\prime}(q=0)=\log (g l) / 2$ does not der $p$ while the $L(q)$ do.

The stability condition implies that the Lyapu ponent is negative. On the other hand, $L(q) \approx q$ los large and positive. So a positive solution for $L\left(q_{*}\right)$ ist and coincides with the condition for a power-lar given for to (6) for $q_{*}=\alpha$. In the Gaussian approx this is seen immediately since in this case

$$
L(q) \approx-\left|\lambda_{2}\right| q+\mu q^{2}
$$

where $\mu$ is the variance of $\lambda_{2}$. In this approximati $\left|\lambda_{2}\right| / \mu$ that makes transparent the fact that fluctuations in the gain have to be of the same order as $\lambda_{2}$ to observe large fluctuations. This is in agreement with the general scenario described in [13].

In Fig 4 we report the generalized Lyapunov exponents and the distributions of the $z_{n}$ variables. The estimated exponents are in very good agreement with the simulations. For instance in the case $p=0.6$ the numerical histogram yield an exponent 0.66 to be compared with the value $q_{*}=0.71 \ldots$

When the condition of stability is violated, some further mechanism of saturation is needed to have a steady distribution. In this case the term $s$ can be ignored. For instance one may consider a nonlinear term in the form of a chaotically-driven logistic map

$$
\left\{\begin{array}{l}
x_{n+1}=f\left(x_{n}\right) \\
E_{n+1}=g\left(x_{n}\right) E_{n}-E_{n}^{2}
\end{array} .\right.
$$

This a particular case of the systems thoroughly studied in [20, 28] and will not thus be considered any further here. In presence of the nonlinear term, $\lambda_{2}$ remains negative and the invariant measure remains broad but has an exponential cutoff at large $E_{n}$. Detailed predictions on the nature of the chaotic intermittency close to the transition can be given [20, 28, 29], including universality of power spectra and Lyapunov exponents. In the next section, we consider an alternative possibility for obtaining steady-state power

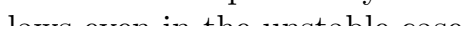
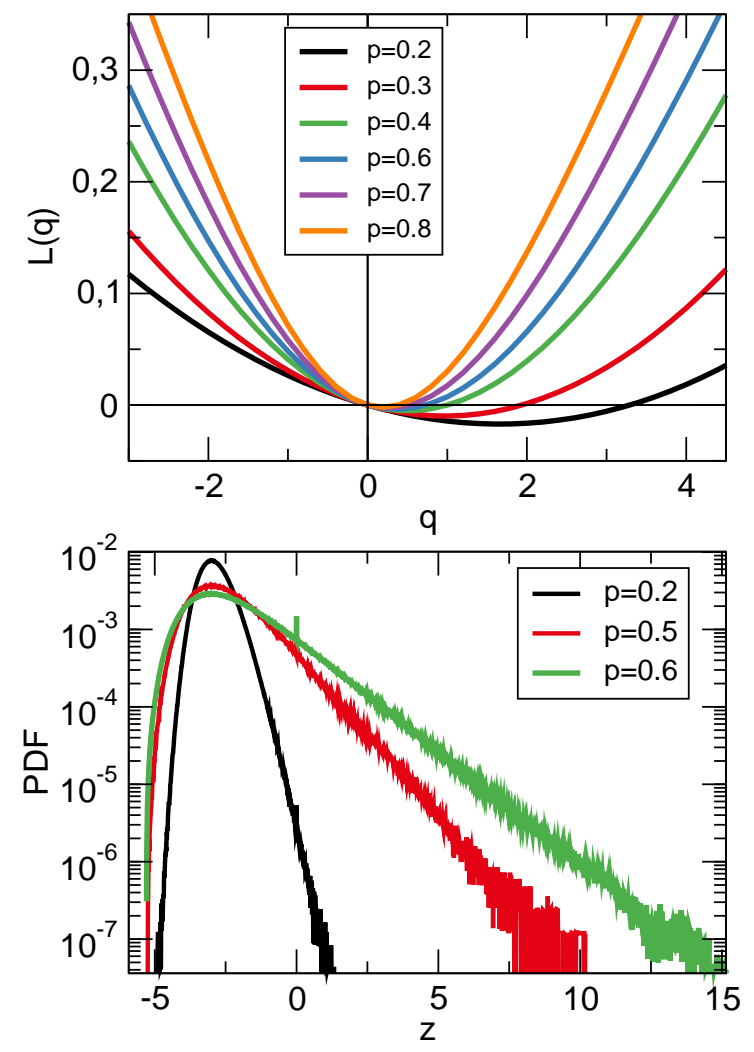

Figure 4: The generalized Lyapunov exponents $L(q)$ for different values of the map parameter $p$ and the distributions of $\log E, g=$ $1.2 l=0.8 s=10^{-3}$. An exponential tail of $P(z) \sim \exp (-\alpha z)$ correspond to a power-law decay $E^{-1-\alpha}$.

\section{Open maps}

We now discuss another possibility to have steady fluctuations with power-law tails namely an open setup where the trajectory are allowed to escape (and be re-injected). The idea is that the distribution of the values of $E_{n}$ is in 
this case determined by the combined effect of the fluctuations of growth rates (as measured by finite-time Lyapunov exponents) and the statistical distribution of the escape events.

Let us consider the growth of a perturbation over a finite time $\tau, E \propto \exp (\lambda(\tau) \tau)$ where $\lambda(\tau)$ is the finite-time Lyapunov exponent [26]. Defining $z=\log E$ its distribution $\mathcal{Q}(z)$ is given by

$$
\mathcal{Q}(z)=\iint d \lambda d \tau \delta(z-\lambda(\tau) \tau) \mathcal{P}(\tau) \mathcal{P}(\lambda, \tau)
$$

basically an average of the growth rates on the distribution of escape times $\mathcal{P}(\tau)$. As usual, for large $\tau$ we introduce the large-deviation function of the form

$$
\mathcal{P}(\lambda, \tau) \sim \exp (-U(\lambda) \tau)
$$

In most cases, the distribution of escape times is Poissonian $\mathcal{P}(\tau)=r \exp (-r \tau)$ where $r$ is the escape rate. Substituting this expression in equation (9), the resulting integra] can be evaluated using the saddle-point approximation: if we denote by $\lambda_{*}$ the saddle point, one obtains the condition $\lambda_{*} U^{\prime}\left(\lambda_{*}\right)-U\left(\lambda_{*}\right)=r$. Then, recalling that the generalized exponents are the Legendre transform of the large-deviation function $L(q)=q \lambda-U(\lambda)$ with $q=U^{\prime}(\lambda)$ one obtains that the asymptotic decay of the distribution

$$
\mathcal{Q}(z) \sim \exp \left(-q_{*} z\right) ; \quad L\left(q_{*}\right)=r
$$

Changing back to the original variable $E$ one obtains again a power-law tail, $E^{-1-q_{*}}$ for large $E$. This last expression generalizes the one given above and confirms that also in the open setup the generalized exponents can be used to estimate the power law decay.

A consequence of the above is that, in the open case we can also consider the unstable case $\lambda_{2}>0$ and expect stationary fat-tailed distributions. Indeed, the equation (11) has a solution $q_{*}$ relatively close to zero. In the Gaussian approximation $L(q)=\lambda_{2} q+\mu q^{2}$ and for small $r$ one has $q_{*} \approx r / \lambda_{2}$. This nicely fits with the estimate given in [5] for observing Lévy fluctuations in amplifying diffusive media with absorbing boundaries, upon identifying $1 / r$ with the average residence time in the medium and $\lambda_{2}$ with the typical amplification time.

To verify the above argument we consider first the simpler case of the map (8) (with $s=0$ ) undergoing a stochastic resetting dynamics. With some preassigned small probability $r$ (which represents the escape rate) the variable $E_{n}$ is reset to some arbitrary value (with no modification on the $x_{n}$ dynamics).

The second case is a deterministic type of resetting, where the $x_{n}$ dynamics is given by the map, see Fig 5

$$
f(x)= \begin{cases}2 x & 0 \leq x \leq 1 / 4 \\ a(x-1 / 4)+\frac{1}{2} & 1 / 4<x \leq 1 / 2 \\ a(x-3 / 4)+\frac{1}{2} & 3 / 4<x \leq 1 \\ 2 x-1 & 3 / 4<x \leq 1\end{cases}
$$

The graph is in Fig 5 along with a sketch of the physical situation where particles are allowed to escape from the graph. For $a>2$ there is escaping region in the interval $(1 / 4-1 / 2 a, 3 / 4-1 / 2 a)$. Thus for $a \rightarrow 2^{+}$the escape rate from the associated chaotic repellor is $r \approx(a-2) / 4$. Whenever the particle trajectory escapes from the unit interval, we reset $E_{n}$ to one and $x_{n}$ to a random uniformly-

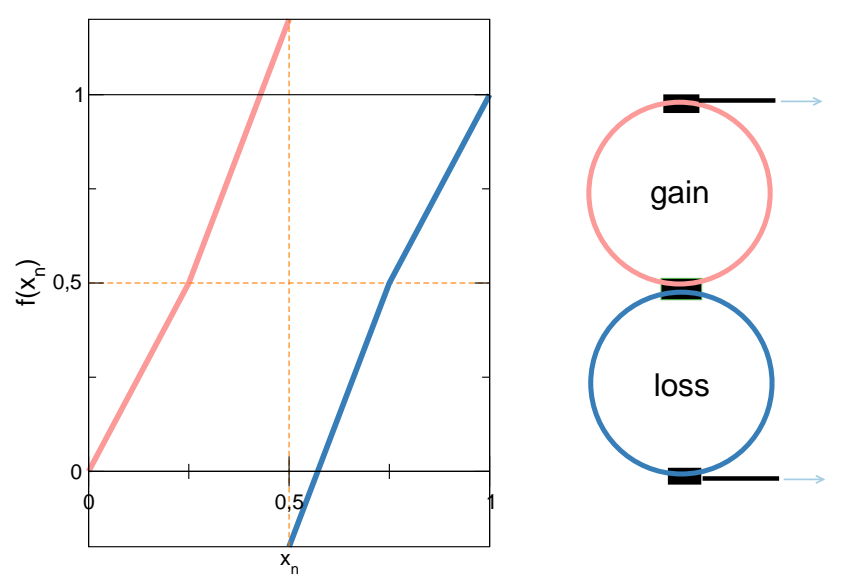

Figure 5: Left: the chaotic map (12) for $a>2$. Right: a sketch of the physical realization the open system in the case of two chaotic cavities connected by a coupler with leaks that allow escape of rays with some probability.

In Fig 6 we considered both examples in the unstable and unstable regimes. The distributions are clearly different from the one of the closed map given in Fig 4. As expected, the deterministic and stochastic case are similar. The generalized Lyapunov exponents as given by formula (7) are also reported. In both cases, the distributions have double-exponential shape with rates in excellent agreement with the one given by (11), represented graphically in the leftmost panels of Fig 6 . In the deterministic case with $a$ not too close from 2 , the escape rate has been estimated numerically.

We conclude with a remark on the finer-scale structure of the distribution. A feature of the model is that the $z_{n}$ variable occurs almost in discrete values. Fig. [7] shows that the distribution has a finer structure with narrow peaks almost equally-spaced (see inset of Fig 7). That should be contrasted with the case of the closed map where $z_{n}$ has continuous values and a smooth distribution.

\section{Conclusions}

Motivated by recent experiments of lasing networks [11, 19], we have introduced a toy map model describing the effect of chaotic diffusion and amplification on a graph structure. Since the motion is purely classical, it should apply when the wavelength is small with respect of the bond lengths. Evidence of large fluctuations and intermittency for the lasing network has been indeed been provided both experimentally and by Monte-Carlo simulation [11]. 

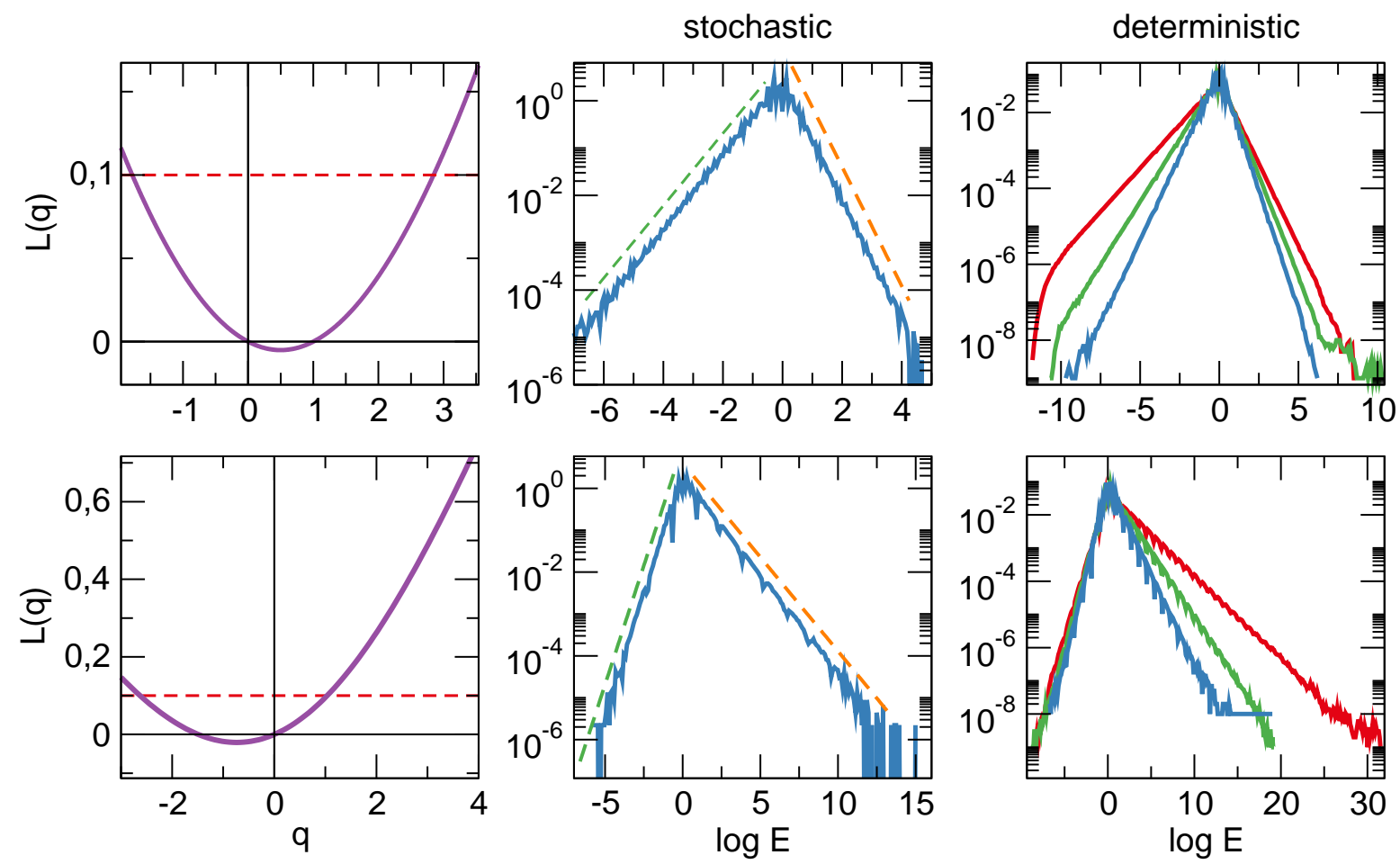

Figure 6: Leftmost panels: the generalized Lyapunov exponents for $p=0.5, l=0.8$, the first row for the stable case $g=1.2$, the second for the unstable one $g=1.4$. Central panels: distributions of the variable $z=\log E$ for the map with stochastic resetting with probability

$r$

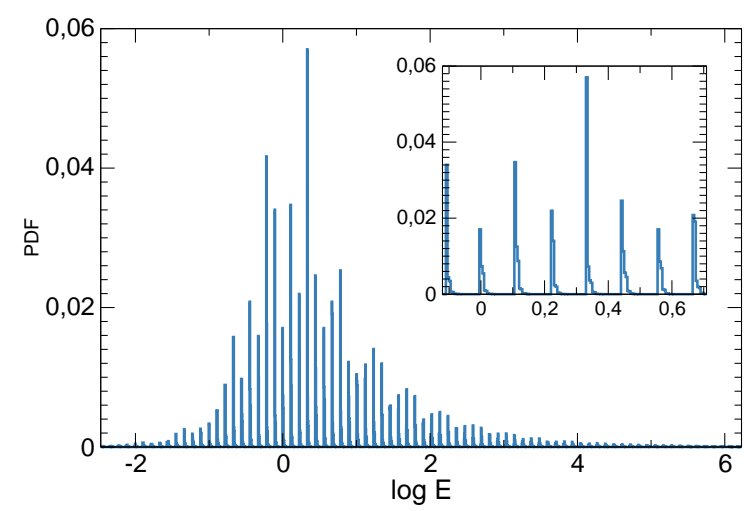

Figure 7: Finer structure of the distribution of $z=\log E$ for the open map (12) with $a=2.4 l=0.8, g=1.4$ (unstable case). The inset shows an enlargement of the central part of the histogram. The peaks are separated by a constant spacing approximatively equal to $\log (g l)$.

Starting from a "Lagrangian" description in terms of chaotic trajectories we derived the corresponding "Eulerian" equations for the probabilities. We discussed the simplest graph, but the generalization to larger graphs is pretty straightforward especially at the level of the master equation (4). In this case the equation can be easily formulated in terms of the transition matrix of the underlying diffusive process and the matrix for stochastic gain or loss terms (see also the Appendix below). by (11). Rightmost panels: distribution of the variable $z=\log E$ for case $a=2.4$ correspond to an escape rate $r \approx 0.083$.

Chaotic diffusion and amplification yield multiplicative fluctuations and power-law steady distributions. In some regimes the variance can diverge leading to Lévy-like statistics. We have confirmed that the Generalized Lyapunov exponents can give a precious hint on the statistics, both in the stable and unstable cases. We have extended this concept to open systems through equation (9) that connects the Generalized exponents with the escape rate. This result should apply under quit general conditions, as demonstrated by the case of random resetting dynamics.

\section{Acknowledgements}

I acknowledge Stefano Gelli for contributing to the initial stage of this work.

\section{Appendix}

In 11] the steady state modes of the lasing networks have been computed using an approach extending the one used for quantum graphs [30]. This is accomplished imposing that a suitable network matrix $N=S P$ has an eigenvalue equal to one. Physically, $S$ is the scattering matrix of the optical couplers (splitters) and $P$ is the so called propagation matrix along the optical fibers and contains both the metric information on the bond length than the gain coefficients [19]. 
To clarify the connection with the map model studied here, let us first consider expressing equation (4) in the "physical" time $t$ (neglecting the term $s$ )

$$
\begin{aligned}
& P_{1}(E, t)=\frac{p}{g} P_{1}\left(\frac{E}{g}, t-T_{1}\right)+\frac{1-p}{l} P_{2}\left(\frac{E}{l}, t-T_{2}\right) \\
& P_{2}(E, t)=\frac{(1-p)}{g} P_{1}\left(\frac{E}{g}, t-T_{1}\right)+\frac{p}{l} P_{2}\left(\frac{E}{l}, t-T_{2}\right)
\end{aligned}
$$

where $T_{1,2}=L_{1,2} / v$ are the travel times (see the footnote in the main text). Taking the Laplace transform in $t$ and introducing the averages

$$
h(E, z)=\int_{0}^{\infty} h(E, t) e^{-z t} d t, \quad I_{1,2} \equiv \int_{0}^{\infty} E P_{1,2}(E, z) d E
$$

one obtains the condition

$$
\left(\begin{array}{l}
I_{1} \\
I_{2}
\end{array}\right)=W G\left(\begin{array}{l}
I_{1} \\
I_{2}
\end{array}\right)
$$

where

$$
W \equiv\left(\begin{array}{cc}
p & 1-p \\
1-p & p
\end{array}\right), G \equiv\left(\begin{array}{cc}
g e^{z T_{1}} & 0 \\
0 & l e^{z T_{2}}
\end{array}\right)
$$

and $W$ is recognized to be the stochastic matrix for a random walk on a graph with two states. To have non-trivial solutions we impose $\operatorname{det}(W G-1)=0$ that determines all possible values of $z$. This equation is obtained by the condition that $N$ has a eigenvalue one, by taking $|N|^{2}$, i.e. the matrix whose elements are the square moduli of it. The stochastic matrix of the graph is thus the square modulus on the scattering matrix $S$ of the coupler $W=|S|^{2}$ while $G=|P|^{2}$. Viewed in this way one can recognize the similarity with the "quantization" procedure outlined in [31, 18] where the classical stochastic transition matrix is replaced by an unitary one describing a quantum map. The generalization of the above to arbitrary graphs is straightforward.

\section{References}

[1] M. A. Porter, J. P. Gleeson, Dynamical Systems on Networks: A Tutorial, Springer series Frontiers in Applied Dynamical Systems: Reviews and Tutorials, Switzerland, 2016.

[2] J. García-Ojalvo, J. Sancho, Noise in spatially extended systems, Springer Verlag, 1999.

[3] A. Crisanti, G. Paladin, A. Vulpiani, Products of random matrices: in Statistical Physics, Vol. 104, Springer Science \& Business Media, 2012.

[4] V. V. Uchaikin, V. M. Zolotarev, Chance and stability: stable distributions and their applications, Walter de Gruyter, 1999.

[5] S. Lepri, S. Cavalieri, G. Oppo, D. S. Wiersma, Statistical regimes of random laser fluctuations, Phys. Rev. A 75 (6) (2007) 063820.

[6] S. Lepri, Fluctuations in a diffusive medium with gain, Phys. Rev. Lett. 110 (2013) 230603.

[7] E. Raposo, A. Gomes, Analytical solution for the Lévy-like steady-state distribution of intensities in random lasers, Phys. Rev. A 91 (4) (2015) 043827.
[8] E. Ignesti, F. Tommasi, L. Fini, S. Lepri, V. Radhalakshmi, D. Wiersma, S. Cavalieri, Experimental and theoretical investigation of statistical regimes in random laser emission, Phys. Rev. A 88 (2013) 033820.

[9] R. Uppu, S. Mujumdar, Lévy exponents as universal identifiers of threshold and criticality in random lasers, Phys. Rev. A 90 (2014) 025801.

[10] A. S. Gomes, E. P. Raposo, A. L. Moura, S. I. Fewo, P. I. Pincheira, V. Jerez, L. J. Maia, C. B. De Araújo, Observation of Lévy distribution and replica symmetry breaking in random lasers from a single set of measurements, Scientific reports 6 (2016) 27987.

[11] S. Lepri, C. Trono, G. Giacomelli, Complex active optical networks as a new laser concept, Physical review letters 118 (12) (2017) 123901.

[12] R. Benzi, G. Paladin, G. Parisi, A. Vulpiani, Characterisation of intermittency in chaotic systems, Journal of Physics A: Mathematical and General 18 (12) (1985) 2157.

[13] J. M. Deutsch, Generic behavior in linear systems with multiplicative noise, Phys. Rev. E 48 (6) (1993) 4179-4182.

[14] S. C. Manrubia, D. H. Zanette, Stochastic multiplicative processes with reset events, Physical Review E 59 (5) (1999) 4945.

[15] S. Gupta, S. N. Majumdar, G. Schehr, Fluctuating interfaces subject to stochastic resetting, Physical review letters 112 (22) (2014) 220601.

[16] R. Klages, Microscopic chaos, fractals and transport in nonequilibrium statistical mechanics, Vol. 24, World Scientific, 2007.

[17] F. Barra, P. Gaspard, Classical dynamics on graphs, Phys. Rev. E 63 (6) (2001) 066215.

[18] P. Pakonski, K. Zyczkowski, M. Kus, Classical 1d maps, quantum graphs and ensembles of unitary matrices, J. Phys. A 34 (43) (2001) 9303.

[19] G. Giacomelli, S. Lepri, C. Trono, Optical networks as complex lasers, Physical Review A 99 (2) (2019) 023841.

20] H. Fujisaka, H. Ishii, M. Inoue, T. Yamada, Intermittency caused by chaotic modulation. ii: Lyapunov exponent, fractal structure and power spectrum, Progress of theoretical physics 76 (6) (1986) 1198-1209.

[21] D. Sornette, R. Cont, Convergent multiplicative processes repelled from zero: power laws and truncated power laws, Journal de Physique I 7 (3) (1997) 431-444.

[22] H. Nakao, Asymptotic power law of moments in a random multiplicative process with weak additive noise, Physical Review E 58 (2) (1998) 1591.

[23] D. Sornette, Multiplicative processes and power laws, Physical Review E 57 (4) (1998) 4811.

[24] M. A. Munoz, T. Hwa, On nonlinear diffusion with multiplicative noise, EPL (Europhysics Letters) 41 (2) (1998) 147.

[25] A. Crisanti, G. Paladin, A. Vulpiani, Generalized Lyapunov exponents in high-dimensional chaotic dynamics and products of large random matrices, Journal of statistical physics 53 (3-4) (1988) 583-601.

[26] A. Pikovsky, A. Politi, Lyapunov exponents: a tool to explore complex dynamics, Cambridge University Press, 2016.

[27] J. Vanneste, Estimating generalized Lyapunov exponents for products of random matrices, Physical Review E 81 (3) (2010) 036701.

[28] T. Yamada, H. Fujisaka, Intermittency caused by chaotic modulation. i: Analysis with a multiplicative noise model, Progress of Theoretical Physics 76 (3) (1986) 582-591.

[29] H. Fujisaka, M. Inoue, Theory of diffusion and intermittency in chaotic systems. iii: New approach to temporal correlations, Progress of theoretical physics 78 (2) (1987) 268-281.

[30] T. Kottos, U. Smilansky, Periodic orbit theory and spectral statistics for quantum graphs, Ann. Phys. 274 (1) (1999) 76124.

[31] G. Tanner, Spectral statistics for unitary transfer matrices of binary graphs, Journal of Physics A: Mathematical and General 33 (18) (2000) 3567. 